P. Galindo-Moreno

A. Fernández-Jiménez

G. Avila-Ortiz

F.J. Silvestre

P. Hernández-Cortés

H.L. Wang

\section{Marginal bone loss around implants placed in maxillary native bone or grafted sinuses: a retrospective cohort study}

\section{Authors' affiliations:}

P. Galindo-Moreno, Department of Oral Surgery and Implant Dentistry, School of Dentistry,

University of Granada, Granada, Spain

A. Fernández-Jiménez, Private practice, Jaén, Spain

G. Avila-Ortiz, Department of Periodontics,

College of Dentistry, University of Iowa, Iowa City, IA, USA

F.J. Silvestre, Department of Stomatology,

University of Valencia, Valencia, Spain

P. Hernández-Cortés, Orthopedic Surgery Unit, San

Cecilio Clinical Hospital, Granada, Spain

H.L. Wang, Department of Periodontics and Oral

Medicine, School of Dentistry, University of

Michigan, Ann Arbor, MI, USA

P. Galindo-Moreno, Department of Periodontics and Oral Medicine, School of Dentistry, University

of Michigan, Ann Arbor, MI, USA

Corresponding author:

Pablo Galindo-Moreno

C/ Recogidas, $39-5^{\circ}$ Izq.

18005 - Granada

Spain.

Tel: +34958 520658

Fax: +34 958520658

e-mail : pgalindo@ugr.es

Key words: alveolar bone loss, bone grafting, bone resorption, dental implants, maxillary sinus

\begin{abstract}
Objectives: To assess differences in marginal bone loss around implants placed in maxillary pristine bone and implants placed following maxillary sinus augmentation over a period of 3 years after functional loading.

Material and methods: Two cohorts of subjects (Group 1: Subjects who received sinus augmentation with simultaneous implant placement; Group 2: Subjects who underwent conventional implant placement in posterior maxillary pristine bone) were included in this retrospective study. Radiographic marginal bone loss was measured around one implant per patient on digitized panoramic radiographs that were obtained the time of prosthesis delivery (baseline) and 12, 24, and 36 months later. The influence of age, gender, smoking habits, history of periodontal disease, and type of prosthetic connection (internal or external) on marginal bone loss was analyzed in function of the type of osseous support (previously grafted or pristine). Results: A total of 105 subjects were included in this study. Cumulative radiographic marginal bone loss ranged from $0 \mathrm{~mm}$ to $3.9 \mathrm{~mm}$ after 36 months of functional loading. There were statistically significant differences in marginal bone loss between implants placed in grafted and pristine bone at the 12-month assessment, but not in the subsequent progression rate. External prosthetic connection, smoking, and history of periodontitis negatively influenced peri-implant bone maintenance, regardless of the type of osseous substrate.

Conclusions: Implants placed in sites that received maxillary sinus augmentation exhibited more marginal bone loss than implants placed in pristine bone, although marginal bone loss mainly occurred during the first 12 months after functional loading. Implants with external implant connection were strongly associated with increased marginal bone loss overtime.
\end{abstract}

Ridge augmentation via bone grafting has become a routine indication to treat alveolar bone deficiencies and facilitate prosthetically driven implant placement. Maxillary sinus floor elevation is not only a predictable surgical procedure to obtain vertical bone augmentation in posterior segments of atrophic maxillae, but also represents an ideal model to investigate healing events following bone grafting (Busenlechner et al. 2009; Price et al. 2011). Clinical, radiographic, and histologic outcomes after maxillary sinus augmentation procedures, applying different grafting materials and surgical techniques, have been extensively reported over the past two decades (Wallace \& Froum 2003; Del Fabbro et al. 2004; Pjetursson et al. 2008; Avila et al. 2010; Galindo-Moreno et al. 2011). A critical clinical question that has attracted the attention of clinicians and researchers is whether implants placed in grafted sites present higher risk of failure than implants placed in native/pristine maxillary bone. According to various systematic reviews, survival rates for implants partially inserted in grafted maxillary sinuses are similar (Wallace \& Froum 2003; Del Fabbro et al. 2004; Pjetursson et al. 2008), or even superior (Olson et al. 2000), to those associated with implants placed in pristine maxillary areas. On the contrary, in a recently published cohort study, it was observed that "...implants placed in augmented sinuses had a lower survival rate compared to implants placed in pristine bone." After a 6-year follow-up period, the mean survival rate for implants placed in 
grafted areas or in native maxillary bone was $86.1 \%$ and $96.4 \%$, respectively (Barone et al. 2011). How can these reported differences be explained?

It is generally accepted that, in order to ensure long-term survival of functionally loaded implants placed in augmented sinuses, tantamount to achieving enough ridge volume for straightforward implant placement is to obtain an osseous substrate which intrinsic structural and physiological characteristics resemble those of native bone. While osseous support of implants placed in pristine maxillae is exclusively constituted by native and newly formed bone, in cases that involve maxillary sinus floor elevation remaining graft particles may also be part of the peri-implant tissue. In the latter, marginal support is provided by a variable amount of native bone, depending on the original remaining alveolar bone height $(\mathrm{RBH})$ (Avila-Ortiz et al. 2012). Finite element analysis studies have suggested that load distribution and marginal bone loss (MBL) around implants placed in grafted sinus cavities may be strongly conditioned by the characteristics of the grafting material (Fanuscu et al. 2004; Huang et al. 2009; Inglam et al. 2010). In this regard, it has been observed that when the grafted volume exhibits less stiffness than the native bone, functional loading produces an increased concomitant stress at the level of the crestal bone (Huang et al. 2009), which is typically associated with MBL (Kitamura et al. 2004). Although finite element analysis studies should be cautiously interpreted, to the light of currently available evidence, it can be hypothesized that implant failure and/or peri-implant bone loss in sites that have undergone sinus floor elevation may be associated with inadequate adaptive responses of the supporting tissues to functional loading. Other factors that have been associated with peri-implant MBL, such as history of periodontal disease (Roccuzzo et al. 2010; Koldsland et al. 2011), smoking (Wallace 2000), and the location of the microgap in function of the type of prosthetic connection (Veis et al. 2010) may play a synergistic role. Hence, the primary objective of this study was to assess differences in MBL around implants placed in maxillary pristine bone and implants placed following maxillary sinus augmentation over a period of 3 years after functional loading. The secondary aim was to evaluate the influence that history of periodontitis, smoking, and type of prosthetic connection have on peri-implant bone resorptive events.

\section{Material and methods}

\section{Study population}

All subjects were selected from a private practice pool following these inclusion criteria: $18-85$ years of age, have at least one implant in the posterior maxillary region with a minimum of 3 years of functional loading, physical status according to the American Society of Anesthesiologists (ASA) I or II, absence of systemic diseases or conditions known to alter bone metabolism, periodontally stable and enrolled in a maintenance program. All records contained standardized orthopantomographs obtained at the time of final restoration delivery (baseline), and at 12, 24, and 36 months after functional loading. Subjects were excluded if they had a history of intake of medications known to modify bone metabolism (e.g., bisphosphonates). Likewise, subjects who developed acute or chronic sinus pathology (i.e., sarcoidosis, osteomas, carcinomas, cancer of any kind, or had postoperative complications related to the procedures described in this study were excluded. The study protocol was reviewed and approved by the ethical committee of the University of Granada for studies involving human subjects.

Consecutive patients that fulfilled the inclusion criteria were elected for this study. The study population was divided into two cohorts of subjects. Subjects were assigned to each cohort based on a sinus augmentation classification that indicates delayed implant placement in clinical scenarios where the (RBH) is $\leq 5 \mathrm{~mm}$ (Wang \& Katranji 2008). The first cohort (Group 1) was formed by subjects who presented $\mathrm{RBH}$ between 5 and $9 \mathrm{~mm}$, which generally allows for maxillary sinus augmentation with simultaneous implant placement. The second cohort (Group 2) included subjects who presented enough alveolar bone height to allow conventional placement of implants with a length of $\geq 12 \mathrm{~mm}$. Subjects received either one of two different implant systems, with internal (Astra Tech AB, Mölndal, Sweden) or external connection (Microdent Implant System, Barcelona, Spain).

\section{Surgical and restorative procedures}

All surgical procedures were conducted under local anesthesia (Ultracain ${ }^{\circledR}$, Aventis Inc., Frankfurt, Germany). In group 1, sinus augmentation procedures were performed following the bone scraper technique as described elsewhere (Galindo-Moreno et al. 2007). Briefly, all sinus cavities were grafted using autologous cortical bone in combination with anorganic bovine bone particles ranging from
250 to $1000 \mu \mathrm{m}$ (Bio-Oss ${ }^{\circledR}$ - Geistlich Pharma AG, Wolhusen, Switzerland) in a $1: 1$ ratio. Prior to bone graft placement, implant osteotomy was performed while protecting the Schneiderian membrane with a blunt metal instrument, according to implant manufacturer's instructions. Then, grafting material was placed to fill the medial half of the sinus cavity, implant/s was/were inserted and the rest of the sinus cavity was filled. A variable volume of bone grafting material, ranging from 3 to $5 \mathrm{cc}$, was used per sinus. An absorbable collagen membrane $\left(\right.$ BioGide $^{\circledR}-$ Geistlich Pharma AG, Wolhusen, Switzerland) was trimmed and adapted over the lateral aspect of the bony window. Soft tissues were approximated and sutured. Primary wound closure was achieved in all cases. In subjects that did not require maxillary sinus augmentation (Group 2), implants were installed following a conventional implant placement protocol. All subjects were asked to comply with a pharmacologic regime that included amoxicillin/clavulanic acid tablets (875/125 mg, TID for 7 days) or, if allergic to penicillin, clindamycin tablets (300 mg, TID for 7 days), as well as antiinflammatory medication (Ibuprofen $600 \mathrm{mg}$, every 4-6 hours as needed to a maximum of $3600 \mathrm{mg}$ per day). Sutures were removed at 2 weeks after sinus surgery (Group 1) or 1 week in belonging to group 2. Subjects were then evaluated at 6-8 weeks intervals, to monitor postoperative healing. Trans-epithelial abutments were placed in a second surgical procedure after a 5-month healing period. Implant-supported prostheses were delivered 4 weeks later. All definitive restorations were screw-retained fixed partial dentures.

Radiographic evaluation of marginal bone loss Standardized digital panoramic radiographs (Kodak ACR-2000, Eastman Kodak Company, Rochester, NY, USA) obtained at the time of final restoration delivery (baseline), and at 12, 24, and 36 months after functional loading were digitized and exported to a computer software for further analysis (Dent-AView v1.0, DigiDent, DIT, Nesher, Israel). To determine MBL, an independent calibrated examiner (A.F-J.) made linear measurements on each panoramic radiograph from the most mesial and distal point of the implant platform to the crestal bone (Figures 1 and 2). Only one implant per subject was analyzed, regardless of the number of implants placed. In order to standardize the measurements and to reduce the influence of anatomical variables, the implant located at 

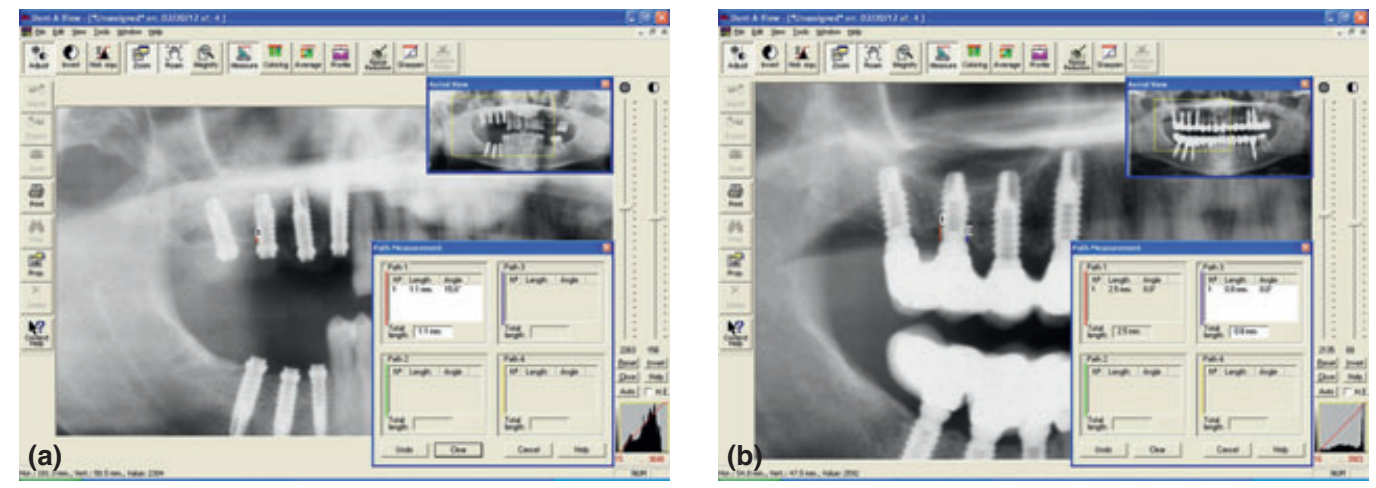

Fig. 1. Radiographic measurements at baseline (a) and 36 months (b) after functional loading in the external connection group
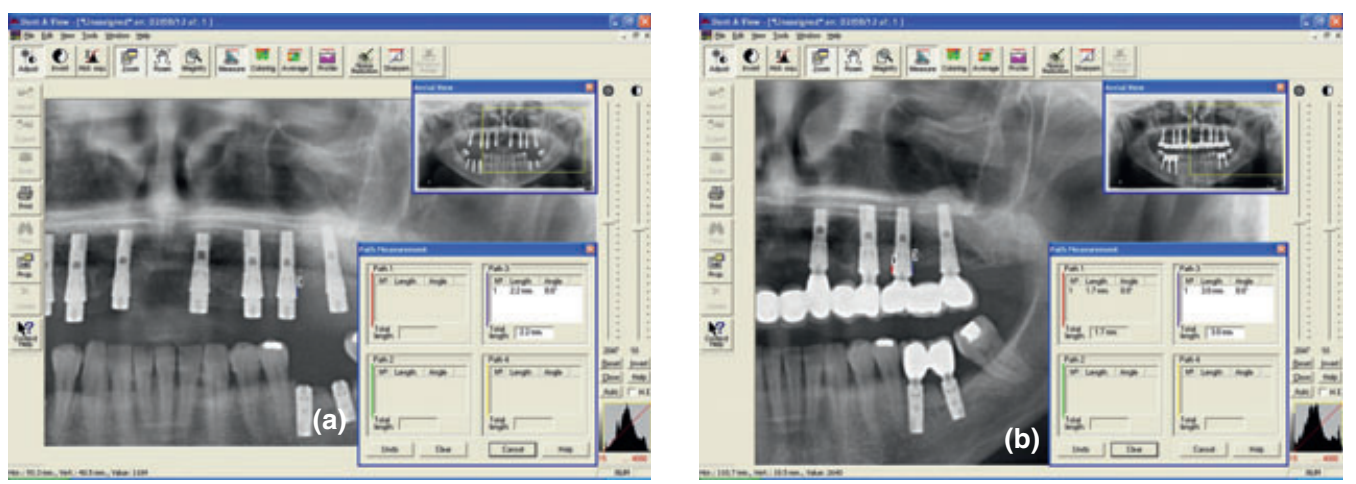

Fig. 2. Radiographic measurements at baseline (a) and 36 months (b) after functional loading in the internal connection group

the site with the shortest $\mathrm{RBH}$ was selected, which most often was the first molar position.

\section{Additional data recorded}

Age, sex, smoking, and drinking habits at the time of enrollment in the study, history of periodontal disease, and type of prosthetic connection (internal or external) were recorded for each subject. Smoking habits were classified using the following criteria: Non-smoker: 0 cigarette/day, mild smoker: 0 -10 cigarette/day, and heavy smoker: $>10$ cigarette/day. Alcohol intake was considered over $10 \mathrm{gr} /$ day (Galindo-Moreno et al. 2005). History of periodontal disease was determined by consulting dental history records. If not available, information was gathered by asking the subject about past dental care and performing a comprehensive periodontal examination.

\section{Statistical analyses}

MBL was expressed as average values (in $\mathrm{mm}$ ) in function of type of bone, interproximal site, time elapsed since functional loading, and type of prosthetic connection. One-way analysis of variance (ANOVA) was performed to identify the categorical vari- ables (gender, history of periodontitis, and smoking) significantly associated with MBL. The relationship between MBL and age was determined by calculating the Pearson correlation coefficient. All these results reported below were robust to the general linear model assumptions. The effect of two variables of interest, type of bone (grafted versus pristine) and type of connection (external versus internal), relative to peri-implant bone loss was analyzed using analysis of covariance (ANCOVA). In this analysis, there were two repeatedmeasures factors: elapsed time since surgery $(12,24$ or 36 months) and location of the marginal bone loss (mesial or distal). The Greenhouse-Geisser correction was used to correct for violation of the sphericity assumption for all decisions in which repeatedmeasures factors with more than 2 levels were involved. All the analyses were carried out using statistical software (SPSS 15, Armonk, NY, USA).

\section{Results}

One hundred and five subjects $174.2 \%$ females) participated in this retrospective cohort study. Age ranged from 32 to 68 years (Mean $=52.37$ years). A total of 46 subjects were enrolled in Group 1 and 59 subjects formed Group 2. Therefore, 105 implants were evaluated. Thirty-three implants in Group 1 had internal connection and 13 had external connection. Twenty-five implants with internal connection and 34 with external connection were allocated in Group 2.

Cumulative radiographic MBL ranged from $0 \mathrm{~mm}$ to $3.9 \mathrm{~mm}$ after 36 months of functional loading. When data from both groups were pooled, $53.3 \%$ of mesial and $49.5 \%$ of distal sites showed $<1 \mathrm{~mm}$ of $\mathrm{MBL}$, while $32.4 \%$ of mesial and $22.9 \%$ of distal sites exhibited no MBL at all. Table 1 shows MBL average values, with the corresponding standard deviation, in function of interproximal site (mesial or distal), elapsed time since functional loading (12, 24 and 36 months), type of prosthetic connection (internal or external), and type of bone (pristine or grafted). Table 2 displays the data that relate to the association between the independent variables and MBL. Independent samples t-tests were used to examine the effects of gender, history of periodontitis, and smoking. Pearson correlation coefficients were computed for age. Smoking independently influenced bone loss during the observed 
Table 1. Average [Medians] values in $\mathrm{mm}$ (standard deviations) for mesial and distal marginal bone loss (intragroup) around implants placed in pristine and grafted bone (intergroup) in function of elapsed time since functional loading (intragroup) and type of prosthetic connection (intergroup)

\begin{tabular}{llllll}
\hline \multirow{2}{*}{ Site } & Bone & Connection & $12 \mathrm{~m}$ & $24 \mathrm{~m}$ & $36 \mathrm{~m}$ \\
\hline Mesial & Pristine & Internal & $0.08[0.01](0.24)$ & $0.11[0.01](0.28)$ & $0.23[0.01](0.51)$ \\
& & External & $0.99[1.09](0.52)$ & $1.16[1.19](0.58)$ & $1.28[1.3](0.63)$ \\
& Grafted & Internal & $0.59[0.01](0.93)$ & $0.71[0.01](0.97)$ & $0.78[0.2](1.02)$ \\
& & External & $1.37[1.11](0.86)$ & $1.51[1.31](0.93)$ & $1.55[1.4](0.91)$ \\
Distal & Pristine & Internal & $0.23[0.01](0.48)$ & $0.27[0.01](0.52)$ & $0.32[0.01](0.54)$ \\
& & External & $1.07[0.91](0.61)$ & $1.27[1.41](0.64)$ & $1.49[1.51](0.71)$ \\
& \multirow{2}{*}{ Grafted } & Internal & $0.74[0.31](0.89)$ & $0.95[0.53](0.93)$ & $1.04[0.51](0.99)$ \\
& & External & $1.09[0.91](0.81)$ & $1.31[1.21](0.88)$ & $1.45[1.51](0.94)$ \\
\hline
\end{tabular}

Table 2. Independent association of marginal bone loss with gender, history of periodontitis, smoking, and age

\begin{tabular}{llllll}
\hline & & Gender & History of periodontitis & Smoking & Age \\
\hline \multirow{2}{*}{$12 \mathrm{~m}$} & Mesial & $0.25[0.70](0.17)$ & $0.41[0.90](0.007)$ & $0.55[1.10](0.001)$ & $0.38(0.001)$ \\
& Distal & $0.24[0.41](0.17)$ & $0.33[0.45](0.028)$ & $0.40[0.50](0.020)$ & $0.26(0.008)$ \\
$24 \mathrm{~m}$ & Mesial & $0.23[0.65](0.23)$ & $0.38[0.90](0.022)$ & $0.53[0.90](0.001)$ & $0.32(0.001)$ \\
& Distal & $0.20[0.60](0.29)$ & $0.29[0.55](0.074)$ & $0.41[0.70](0.020)$ & $0.25(0.009)$ \\
\multirow{2}{*}{$36 \mathrm{~m}$} & Mesial & $0.19[0.52](0.34)$ & $0.36[1.05](0.039)$ & $0.47[1.05](0.002)$ & $0.30(0.002)$ \\
& Distal & $0.20[0.57](0.32)$ & $0.29[0.65](0.110)$ & $0.40[0.90](0.030)$ & $0.28(0.004)$ \\
\hline
\end{tabular}

Differences between averages [Medians], marginal bone loss for gender (females-males), periodontitis (periodontal vs. non-periodontal), and Smoking (smokers vs. non-smokers). Independent samples $t$-test $P$-values are between parenthesis. Last column, Pearson correlation coefficients for age ( $p$-values between parenthesis).

times at both mesial and distal sites. Likewise, an association between history of periodontitis and increased MBL was observed, except on distal sites at 24 and 36 months. Increased MBL was also associated with older age.

The 2 (intergroup, type of bone: pristine vs. grafted) by 2 (intergroup, type of connection: external vs. internal) by 3 (intragroup, Times: 12, 24, and 36 months) by 2 (intragroup, Sites: mesial vs. distall repeatedmeasures ANCOVA, using history of periodontitis, smoking and age as covariates, revealed that peri-implant MBL was higher in grafted $(1.09 \mathrm{~mm})$ than in pristine $(0.71 \mathrm{~mm})$ bone $[\mathrm{F} \quad(1,98)=5.62, \quad \mathrm{p}=0.02]$. MBL progression rate was not different between both groups. Interestingly, MBL was significantly higher around implants with external $(1.30 \mathrm{~mm})$ than with internal $(0.50 \mathrm{~mm}) \quad$ connections $\quad[F(1,98)=17.23$, $P<0.01]$. The type of connection by elapsed time interaction was also significant $[F$ $(2,196)=4.85, P<0.01]$. Trend analyses of this interaction showed that MBL was steeper overtime for the external than the internal connection implants $[F(1,98)=5.51, \quad P=0.02$ (Slopes were $0.18 \mathrm{~mm} /$ year and $0.075 \mathrm{~mm}$ / year, respectively)]. No other significant effects were observed. bone, reported by Barone and collaborators (Barone et al. 2011). Differences in the biomechanical and biological properties of the tissue that directly interfaces with the implant surface may be the main reason for variations in MBL patterns noticed between both groups in the present study. The effect of biomechanical adaptive responses after functional loading on progressive $\mathrm{MBL}$ in the sinus augmentation model has been investigated in several finite element analysis studies. Cehreli and collaborators reported that, although sinus augmentation normally results in more vertical bone support, it also gives rise to the appearance of strains in the sinus floor region, at the boundary between the native and the neoformed tissue (Cehreli et al. 2007). In this regard, Inglam et al. noticed that when the stiffness of the grafted area is less than that of the cancellous bone high-level strain is primarily distributed at the crestal level, which may promote MBL. Therefore, grafted areas should ideally have certain stiffness (similar or superior to adjacent native bone), so efficient loading forces distribution can be reached due to the exhibited similar values of strain energy density in the crestal cortical, cancellous, and grafted bone (Inglam et al. 2010). In this study, a composite graft (autologous cortical bone and anorganic bovine bone) was used for maxillary sinus augmentation. Physical properties of anorganic bovine bone $(\mathrm{ABB})$ are comparable with human bone, given their similarities in both crystalline and morphological structure. Compared with normal human cancellous bone, $\mathrm{ABB}$ has a slightly higher modulus of elasticity [11 GPa] (Yildirim et al. 2000) and a similar compressive strength of $35 \mathrm{MPa}$ (Scarano et al. 2006). It has been shown that in augmented sinus areas, bovine bone material behaves like autologous chin bone particles, although ABB has a much slower resorption rate than autogenous grafts (Sbordone et al. 2011). Hence, the differences between the used biomaterials were minimal, and no complication that could have affected graft consolidation was noticed during the observational period, but still MBL was higher around implants placed in grafted areas. This indicates that despite careful patient and biomaterial selection, the sequence of healing events following maxillary sinus augmentation may not always lead to obtain implant-supporting tissues with optimal properties. This is possibly related to variations in maturation and consolidation of the grafted area (i.e., reduced stiffness). 
In the present study, smoking and history of periodontitis negatively influenced $\mathrm{MBL}$ with statistical significance regardless of the type of osseous substrate (grafted or pristine). Nonetheless, this is not surprising because both variables have been reported to play an important detrimental role in the maintenance of peri-implant crestal bone. Multiple studies have demonstrated an increased risk of MBL for smokers compared with nonsmokers, with odds ratio of peri-implantitis in smokers that range from 3.6 to 4.6 /Galindo-Moreno et al. 2005; Nitzan et al. 2005; Heitz-Mayfield \& Huynh-Ba 2009). Former smokers also present more MBL when compared with non-smokers (Levin 2008). In a recent meta-analysis, MBL in subjects with history of periodontitis was found to be higher than in periodontally healthy subjects [mean difference $=0.61 \mathrm{~mm}] \quad$ (Safii et al. 2010). It has also been shown that the combination of history of periodontitis and smoking increases the risk of peri-implant bone loss (Feloutzis et al. 2003; Wennstrom et al. 2004; Heitz-Mayfield \& Huynh-Ba 2009). A recent study has reported that after 10 years, implants placed in tobacco smokers with a history of treated periodontitis yielded higher marginal bone loss compared with implants placed in periodontally healthy smokers, independent of the implant system used (Aglietta et al. 2011). Another study showed that implants in periodontally compromised, but non-smoking subjects who were previously treated for periodontitis had a tendency to exhibit more MBL when compared to those placed in periodontally healthy subjects (Matarasso et al. 2010). Confounding factors between these two variables could be argued because it is evident that tobacco smokers are more prone to develop periodontitis than non-smokers (Heitz-Mayfield 2005). In our study, after analyzing the effect of each variable, tobacco appeared to play a more determinant role in the progression of $\mathrm{MBL}$ overtime as compared to the other variables $(P<0.0001)$.

Interestingly, the variable that showed the strongest association with MBL in this study was the type of prosthetic connection. MBL

\section{References}

Abrahamsson, I. \& Berglundh, T. (2009) Effects of different implant surfaces and designs on marginal bone-level alterations: a review. Clinical Oral Implants Research 20(Suppl 4): $207-$ 215.

Aglietta, M., Siciliano, V.I., Rasperini, G., Cafiero, C., Lang, N.P. \& Salvi, G.E. (2011) A 10-year was higher around implants with external connection than around those with internal prosthetic connection, regardless of bone type (grafted or non-grafted). These differences were sustained over the 36-month observational period. As of 2007, of all dental implant systems presently available in the market, only three had scientific documentation on periimplant MBL reported in two or more 5-year prospective clinical studies (Laurell \& Lundgren 2011). These systems showed mean marginal bone loss values over 5 years well below what is hitherto accepted as success (Misch et al. 2008). In the majority of cases, most of the cumulative MBL takes place at early stages, particularly during the interval between abutment connection and crown delivery (Cardaropoli et al. 2006). It was suggested that this phenomena occurs because of the establishment of a peri-implant biological width (Berglundh \& Lindhe 1996; Oh et al. 2002). However, it can be inferred that this physiological event does not happen to the same degree around all implants, and it may occur at different points in time. This notion is in accordance with our findings, where external prosthetic connection was associated with increased MBL, in particular during the first 12 months after functional loading. Although strongly significant, the reason for the difference in MBL between systems should be speculated upon a wide array of subjectrelated factors such as implant-supporting bone features (location, nature, or architecture), microbiologic characteristics, and individual inflammatory profiles; implant-related factors such as surface (Abrahamsson \& Berglundh 2009), macro- (Hansson 2000; Novaes et al. 2006), or micro-design (Hansson \& Werke 2003), roughness at the cervical portion (Hansson \& Norton 1999; Aloy-Prosper et al. 2011), platform switching (Vela-Nebot et al. 2006; Canullo et al. 2010; Serrano-Sanchez et al. 2011) and location of the micro-gap (Piattelli et al. 2003; Dibart et al. 2005); or surgical-related factors such as distance between implants (Tarnow et al. 2000; Traini et al. 2010) and delayed versus immediate placement (Herzberg et al. 2006). Future studies should be conducted in order to elucidate the effect of this plethora of variables on MBL to better understand these phenomena and prevent its appearance.

Despite the efforts made by the investigators to comply with high standards of research quality, this study presents some limitations. First, number of subjects and implants are not equally distributed per group. Also, obtaining radiographic $\mathrm{MBL}$ measurements from cone beam computer tomographic (CBCT) scans would have provided more accuracy and the possibility of performing a tridimensional analysis. However, this method was not part of this study due to unavailability in existing dental records.

\section{Conclusions}

Implants placed in sites that received maxillary sinus augmentation exhibited more MBL than implants placed in pristine bone, although MBL mainly occurred during the first 12 months after functional loading. Smoking and history of periodontitis negatively influenced MBL with statistical significance regardless of the type of osseous substrate. Implants with external implant connection were strongly associated with increased MBL overtime.

Acknowledgements: The authors would like to thank Dr Andres Catena for his support as a statistical advisor and Dr O ' Valle for his valuable contribution in the design of this study.

This study was partially supported by Junta de Andalucía Funding Program for research groups in Spain (Projects \#CTS-138 and \#CTS-583).

\section{Disclosure}

Authors do not have any financial interests, either directly or indirectly, in the products listed in the study.

retrospective analysis of marginal bone-level changes around implants in periodontally healthy and periodontally compromised tobacco smokers. Clinical Oral Implants Research 22: 47-53.

Aloy-Prosper, A., Maestre-Ferrin, L., Penarrocha-O1tra, D. \& Penarrocha-Diago, M. (2011) Marginal bone loss in relation to the implant neck surface: an update. Medicina Oral, Patología Oral y Cirugía Bucal 16: e365-e368.

Avila, G., Wang, H.L., Galindo-Moreno, P., Misch, C.E., Bagramian, R.A., Rudek, I., Benavides, E., Moreno-Riestra, I., Braun, T. \& Neiva, R. (2010) The influence of the bucco-palatal distance on 
sinus augmentation outcomes. Journal of Periodontology 81: 1041-1050.

Avila-Ortiz, G., Neiva, R., Galindo-Moreno, P., Rudek, I., Benavides, E. \& Wang, H.L. (2012) Analysis of the influence of residual alveolar bone height on sinus augmentation outcomes. Clinical Oral Implants Research 23: 1082-1088.

Barone, A., Orlando, B., Tonelli, P. \& Covani, U. (2011) Survival rate for implants placed in the posterior maxilla with and without sinus augmentation: a comparative cohort study. Journal of Periodontology 82: 219-226.

Berglundh, T. \& Lindhe, J. (1996) Dimension of the periimplant mucosa. Biological width revisited. Journal of Clinical Periodontology 23: 971-973.

Busenlechner, D., Huber, C.D., Vasak, C., Dobsak, A., Gruber, R. \& Watzek, G. (2009) Sinus augmentation analysis revised: the gradient of graft consolidation. Clinical Oral Implants Research 20: 1078-1083.

Canullo, L., Fedele, G.R., Iannello, G. \& Jepsen, S. (2010) Platform switching and marginal bonelevel alterations: the results of a randomizedcontrolled trial. Clinical Oral Implants Research 21: $115-121$

Cardaropoli, G., Lekholm, U. \& Wennstrom, J.L. (2006) Tissue alterations at implant-supported single-tooth replacements: a 1-year prospective clinical study. Clinical Oral Implants Research 17: 165-171.

Cehreli, M.C., Akkocaoglu, M., Comert, A., Tekdemir, I. \& Akca, K. (2007) Bone strains around apically free versus grafted implants in the posterior maxilla of human cadavers. Medical and Biological Engineering and Computing 45: 395-402.

Del Fabbro, M., Testori, T., Francetti, L. \& Weinstein, R. (2004) Systematic review of survival rates for implants placed in the grafted maxillary sinus. International Journal of Periodontics and Restorative Dentistry 24: 565-577.

Dibart, S., Warbington, M., Su, M.F. \& Skobe, Z. (2005) In vitro evaluation of the implant-abutment bacterial seal: the locking taper system. International Journal of Oral and Maxillofacial Implants 20: 732-737.

Fanuscu, M.I., Vu, H.V. \& Poncelet, B. (2004) Implant biomechanics in grafted sinus: a finite element analysis. Journal of Oral Implantology 30: 59-68.

Feloutzis, A., Lang, N.P., Tonetti, M.S., Burgin, W., Bragger, U., Buser, D., Duff, G.W. \& Kornman, K.S. (2003) IL-1 gene polymorphism and smoking as risk factors for peri-implant bone loss in a well-maintained population. Clinical Oral Implants Research 14: 10-17.

Galindo-Moreno, P., Avila, G., Fernandez-Barbero, J.E., Aguilar, M., Sanchez-Fernandez, E., Cutando, A. \& Wang, H.L. (2007) Evaluation of sinus floor elevation using a composite bone graft mixture. Clinical Oral Implants Research 18: 376-382.

Galindo-Moreno, P., Fauri, M., Avila-Ortiz, G., Fernandez-Barbero, J.E., Cabrera-Leon, A. \& Sanchez-Fernandez, E. (2005) Influence of alcohol and tobacco habits on peri-implant marginal bone loss: a prospective study. Clinical Oral Implants Research 16: 579-586.

Galindo-Moreno, P., Moreno-Riestra, I., Avila, G., Padial-Molina, M., Paya, J.A., Wang, H.L.
\& O’Valle, F. (2011) Effect of anorganic bovine bone to autogenous cortical bone ratio upon bone remodeling patterns following maxillary sinus augmentation. Clinical Oral Implants Research 22: 857-864.

Hansson, S. (2000) Implant-abutment interface: biomechanical study of flat top versus conical. Clinical Implant Dentistry and Related Research 2: 33-41.

Hansson, S. \& Norton, M. (1999) The relation between surface roughness and interfacial shear strength for bone-anchored implants. A mathematical model. Journal of Biomechanics 32: 829-836.

Hansson, S. \& Werke, M. (2003) The implant thread as a retention element in cortical bone: the effect of thread size and thread profile: a finite element study. Journal of Biomechanics 36: 1247-1258.

Heitz-Mayfield, L.J. (2005) Disease progression: identification of high-risk groups and individuals for periodontitis. Journal of Clinical Periodontology 32(Suppl 6): 196-209.

Heitz-Mayfield, L.J. \& Huynh-Ba, G. (2009) History of treated periodontitis and smoking as risks for implant therapy. International Journal of Oral and Maxillofacial Implants 24(Suppl): 39-68.

Herzberg, R., Dolev, E. \& Schwartz-Arad, D. (2006) Implant marginal bone loss in maxillary sinus grafts. International Journal of Oral and Maxillofacial Implants 21: 103-110.

Huang, H.L., Fuh, L.J., Ko, C.C., Hsu, J.T. \& Chen, C.C. (2009) Biomechanical effects of a maxillary implant in the augmented sinus: a three-dimensional finite element analysis. International Journal of Oral and Maxillofacial Implants 24: 455-462.

Inglam, S., Suebnukarn, S., Tharanon, W., Apatananon, T. \& Sitthiseripratip, K. (2010) Influence of graft quality and marginal bone loss on implants placed in maxillary grafted sinus: a finite element study. Medical and Biological Engineering and Computing 48: 681-689.

Johansson, B., Wannfors, K., Ekenback, J., Smedberg, J.I. \& Hirsch, J. (1999) Implants and sinusinlay bone grafts in a 1-stage procedure on severely atrophied maxillae: surgical aspects of a 3-year follow-up study. International Journal of Oral and Maxillofacial Implants 14: 811-818.

Kitamura, E., Stegaroiu, R., Nomura, S. \& Miyakawa, O. (2004) Biomechanical aspects of marginal bone resorption around osseointegrated implants: considerations based on a three-dimensional finite element analysis. Clinical Oral Implants Research 15: 401-412.

Koldsland, O.C., Scheie, A.A. \& Aass, A.M. (2011) The association between selected risk indicators and severity of peri-implantitis using mixed model analyses. Journal of Clinical Periodontology 38: 285-292.

Laurell, L. \& Lundgren, D. (2011) Marginal bone level changes at dental implants after 5 years in function: a meta-analysis. Clinical Implant Dentistry and Related Research 13: 19-28.

Levin, L. (2008) Dealing with dental implant failures. Journal of Applied Oral Science : Revista FOB 16: 171-175.

Matarasso, S., Rasperini, G., Iorio Siciliano, V., Salvi, G.E., Lang, N.P. \& Aglietta, M. (2010) A 10-year retrospective analysis of radiographic bone-level changes of implants supporting singleunit crowns in periodontally compromised vs. periodontally healthy patients. Clinical Oral Implants Research 21: 898-903.

Misch, C.E., Perel, M.L., Wang, H.L., Sammartino, G., Galindo-Moreno, P., Trisi, P., Steigmann, M., Rebaudi, A., Palti, A., Pikos, M.A., SchwartzArad, D., Choukroun, J., Gutierrez-Perez, J.L., Marenzi, G. \& Valavanis, D.K. (2008) Implant success, survival, and failure: the International Congress of Oral Implantologists (ICOI) Pisa Consensus Conference. Implant Dentistry 17: 5-15.

Nitzan, D., Mamlider, A., Levin, L. \& SchwartzArad, D. (2005) Impact of smoking on marginal bone loss. International Journal of Oral and Maxillofacial Implants 20: 605-609.

Novaes, A.B. Jr, Papalexiou, V., Muglia, V. \& Taba, M. Jr (2006) Influence of interimplant distance on gingival papilla formation and bone resorption: clinical-radiographic study in dogs. International Journal of Oral and Maxillofacial Implants 21: 45-51.

Oh, T.J., Yoon, J., Misch, C.E. \& Wang, H.L. (2002) The causes of early implant bone loss: myth or science? Journal of Periodontology 73: 322-333.

Olson, J.W., Dent, C.D., Morris, H.F. \& Ochi, S. (2000) Long-term assessment (5 to 71 months) of endosseous dental implants placed in the augmented maxillary sinus. Annals of Periodontology 5: 152-156.

Piattelli, A., Degidi, M., Paolantonio, M., Mangano, C. \& Scarano, A. (2003) Residual aluminum oxide on the surface of titanium implants has no effect on osseointegration. Biomaterials 24: 4081-4089.

Pjetursson, B.E., Tan, W.C., Zwahlen, M. \& Lang, N.P. (2008) A systematic review of the success of sinus floor elevation and survival of implants inserted in combination with sinus floor elevation. Journal of Clinical Periodontology 35: 216-240.

Price, A.M., Nunn, M., Oppenheim, F.G. \& Van Dyke, T.E. (2011) De novo bone formation after the sinus lift procedure. Journal of Periodontology 82: 1245-1255.

Roccuzzo, M., De Angelis, N., Bonino, L. \& Aglietta, M. (2010) Ten-year results of a three-arm prospective cohort study on implants in periodontally compromised patients. Part 1: implant loss and radiographic bone loss. Clinical Oral Implants Research 21: 490-496.

Safii, S.H., Palmer, R.M. \& Wilson, R.F. (2010) Risk of implant failure and marginal bone loss in subjects with a history of periodontitis: a systematic review and meta-analysis. Clinical Implant Dentistry and Related Research 12: 165-174.

Sbordone, L., Levin, L., Guidetti, F., Sbordone, C., Glikman, A. \& Schwartz-Arad, D. (2011) Apical and marginal bone alterations around implants in maxillary sinus augmentation grafted with autogenous bone or bovine bone material and simultaneous or delayed dental implant positioning. Clinical Oral Implants Research 22: 485-491.

Scarano, A., Degidi, M., Iezzi, G., Pecora, G., Piattelli, M., Orsini, G., Caputi, S., Perrotti, V., Mangano, C. \& Piattelli, A. (2006) Maxillary 
sinus augmentation with different biomaterials: a comparative histologic and histomorphometric study in man. Implant Dentistry 15: 197-207.

Serrano-Sanchez, P., Calvo-Guirado, J.L., Manzanera-Pastor, E., Lorrio-Castro, C., Bretones-Lopez, P. \& Perez-Llanes, J.A. (2011) The influence of platform switching in dental implants. A literature review. Medicina Oral, Patología Oral y Cirugía Bucal 16: e400-e405.

Tarnow, D.P., Cho, S.C. \& Wallace, S.S. (2000) The effect of inter-implant distance on the height of inter-implant bone crest. Journal of Periodontology 71: 546-549.

Traini, T., Novaes, A.B., Piattelli, A., Papalexiou, V. \& Muglia, V.A. (2010) The relationship between interimplant distances and vascularization of the interimplant bone. Clinical Oral Implants Research 21: 822-829.
Veis, A., Parissis, N., Tsirlis, A., Papadeli, C., Marinis, G. \& Zogakis, A. (2010) Evaluation of peri-implant marginal bone loss using modified abutment connections at various crestal level placements. International Journal of Periodontics and Restorative Dentistry 30: 609-617.

Vela-Nebot, X., Rodriguez-Ciurana, X., RodadoAlonso, C. \& Segala-Torres, M. (2006) Benefits of an implant platform modification technique to reduce crestal bone resorption. Implant Dentistry 15: 313-320.

Wallace, R.H. (2000) The relationship between cigarette smoking and dental implant failure. European Journal of Prosthodontics and Restorative Dentistry 8: 103-106.

Wallace, S.S. \& Froum, S.J. (2003) Effect of maxillary sinus augmentation on the survival of endosseous dental implants. A systematic review. Annals of Periodontology 8: 328-343.
Wang, H.L. \& Katranji, A. (2008) ABC sinus augmentation classification. International Journal of Periodontics and Restorative Dentistry 28: 383-389.

Wennstrom, J., Zurdo, J., Karlsson, S., Ekestubbe, A., Grondahl, K. \& Lindhe, J. (2004) Bone level change at implant-supported fixed partial dentures with and without cantilever extension after 5 years in function. Journal of Clinical Periodontology 31: 1077-1083.

Yildirim, M., Spiekermann, H., Biesterfeld, S. \& Edelhoff, D. (2000) Maxillary sinus augmentation using xenogenic bone substitute material Bio-Oss in combination with venous blood. A histologic and histomorphometric study in humans. Clinical Oral Implants Research 11: 217-229. 